\title{
Strain elastography and lymph nodes
}

\author{
Mehmet B. Çildağ \\ Adnan Menderes University, Turkey
}

I read with interest the currently published article written by Özel et al. [1] entitled "Evaluation of diagnostic value of conventional and color Doppler ultrasound with elastography strain ratios in differentiation between benign and malignant lymph nodes". They found elastography strain (ESR) ratios to be higher in malignant lymph nodes (3.93, mean SD [3.71]) than benign lymph nodes (2.94, mean SD [3.61]). However, the difference was not statistically significant. Real-time elastography has shown potential diagnostic value in many diseases. However, elastography has not been used as a routine examination in the evaluation of lymph nodes. There were many studies about usage of elastography in lymph nodes [2-4]. In these studies, different elastographic methods such as strain elastography or shear wave elastography, as well as different elasticity assessments such as visual scoring or comparing strain ratio values with reference tissue, were used. In this study, Özel et al. [1] compared strain ratio values with reference tissue; in this method the strain in the region of interest (ROI) can be compared to an ROI in the reference tissue that is experiencing similar stress, and this provides a semi-quantitative measurement of the relative rather than absolute tissue stiffness. In this study the distribution of lymph nodes regions were very heterogenous, such as neck, axillary, and inguinal. As mentioned in the study, the strain ratio values were affected by the adjacent tissues and were highly user-dependent, which is a limitation of this method. In this study, as I understand it, muscles were used as the reference tissue, which may be usable in the neck region. But how we do use muscles as a reference tissue in the axillary region; maybe surrounding fat tissue is usable in the axillary region. However, I think that the regional comparison of lymph node strain ratio values may be better in such cases.

\section{Conflict of interest}

The author reports no conflict of interest.

\section{References}

1. Özel D, Özel B. Evaluation of diagnostic value of conventional and color Doppler ultrasound with elastography strain ratios in differentiation between benign and malignant lymph nodes. Pol J Radiol 2018; 83: 32-36.

2. Park YM, Fornage BD, Benveniste AP, et al. Strain elastography of abnormal axillary nodes in breast cancer patients does not improve diagnostic accuracy compared with conventional ultrasound alone. Am J Roentgenol 2014; 203: 1371-1378.
3. Ying M, Bhatia KSS, Lee YH, et al. Review of ultrasonography of malignant neck nodes: Greyscale, Doppler, contrast enhancement and elastography. Cancer Imaging 2014; 13: 658-669.

4. Desmots F, Fakhry N, Mancini J, et al. Shear wave elastography in head and neck lymph node assessment: image quality and diagnostic impact compared with B-mode and doppler ultrasonography. Ultrasound Med Biol 2016; 42: 387-398. 